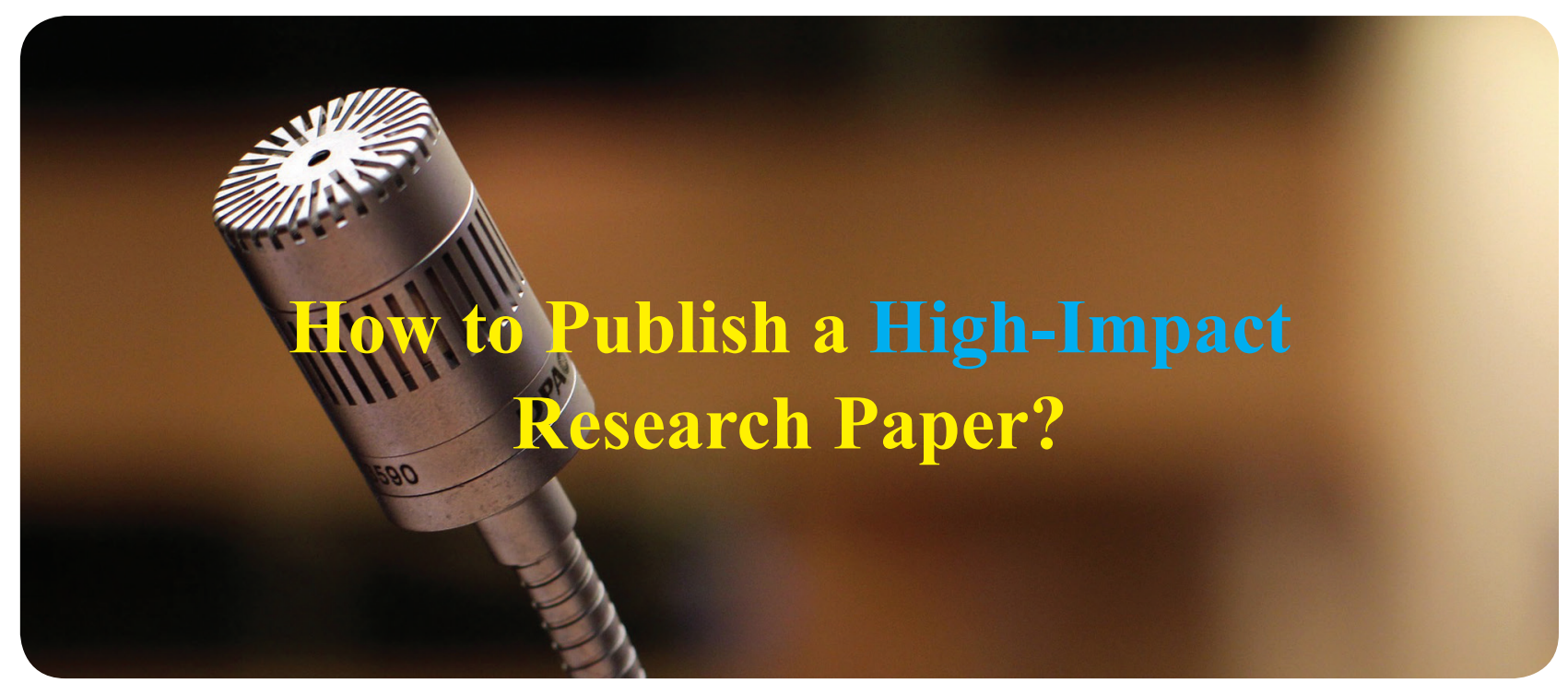

\section{ISMRP 背景介绍}

2018 年 9 月 15-16日, 为期两天的 “第二届国际 医学研究与发表高峰论坛暨首届国际医学前沿研讨 会” 在江城武汉成功举办。

本次高峰论坛由华誉出版社 (Xia \& He Publishing Inc.) 和华中科技大学同济医学院《当代医学科 学》 (Current Medical Science) 杂志社联合主办, 武汉美捷登生物科技有限公司承办, 同时得到了同 济医学院海外校友总会、中国英文科技论文编辑联 盟、武汉华易研生物科技有限公司等机构, 以及新 华社《半月谈》、湖北电视台、南方都市报、同济大 健康等媒体的大力支持。

本届峰会的口号是 “做真实研究、出创新成果、 发高质文章、办优秀期刊”, 旨在为业界专家和医学 工作者相互学习、相互交流、相互协作，搭建了一个 高水平、高规格的沟通平台, 拓宽学术视野, 提升 医学研究与发表水平, 共同探讨如何打造中国具有 国际影响力的学术期刊等问题。

\section{赵剑飞个人简介}

《NEJM 医学前沿》杂志副主编, 全面负责杂志 出版方面的相关运作, 包括内容把控和培训课程策 划等。《NEJM 医学前沿》是《新英格兰医学杂志》 (New England Journal of Medicine, NEJM) 出版方 NEJM 集团推出的 NEJM精选中文版, 出版发表于 NEJM和 NEJM Journal Watch 的重量级论文的全 文中译, 涵盖 $N E J M$ 发表的所有领域, 部分译文附
有针对中国临床实践的述评。此前, 赵剑飞博士在自 然杂志社旗下的《自然通讯》(Nature Communications) 担任专职科学编辑, 审阅来自世界各国的表观 遗传学等生物领域论文。作为自然杂志社首位落地中 国的生命科学领域编辑, 与中国生命科学界的科研 人员建立了密切联系。赵剑飞博士本科毕业于北京 大学生命科学学院, 在美国俄勒冈大学获得博士学 位, 随后在美国国立癌症研究所完成了博士后研究, 专注于表观遗传因素对基因表达调控的影响。

\section{以下是《医学研究与发表》对赵剑飞的专访}

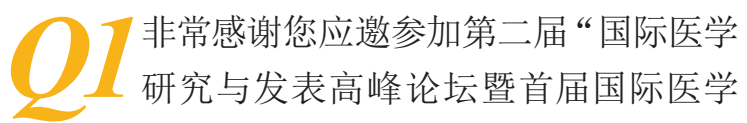
前沿研讨会”, 请问您认为本次峰会与其他学术会议 相比，有哪些不同和重要意义?

$A 7$ 首先非常感谢夏主编, 受邀美捷登 递 NEJM 医学前沿的一些知识。此次参会, 亦是代 表 NEJM 医学前沿的主编、New England Journal of Medicine的副主编——肖瑞平老师。我对此次峰会 印象深刻的是, 它的实操性特别强, 目的很明确, 向大家教授如何将文章发表到高水平的医学期刊上。 如 15 日下午开展的讲座中, 华中科技大学流行病学 系主任魏老师讲授了如何去做临床研究, 16 日上午 Gut主编就 “如何在 Gut杂志上发表文章” 发表了 演讲。我觉得对于广大医生或医学生, 这样的讲座 


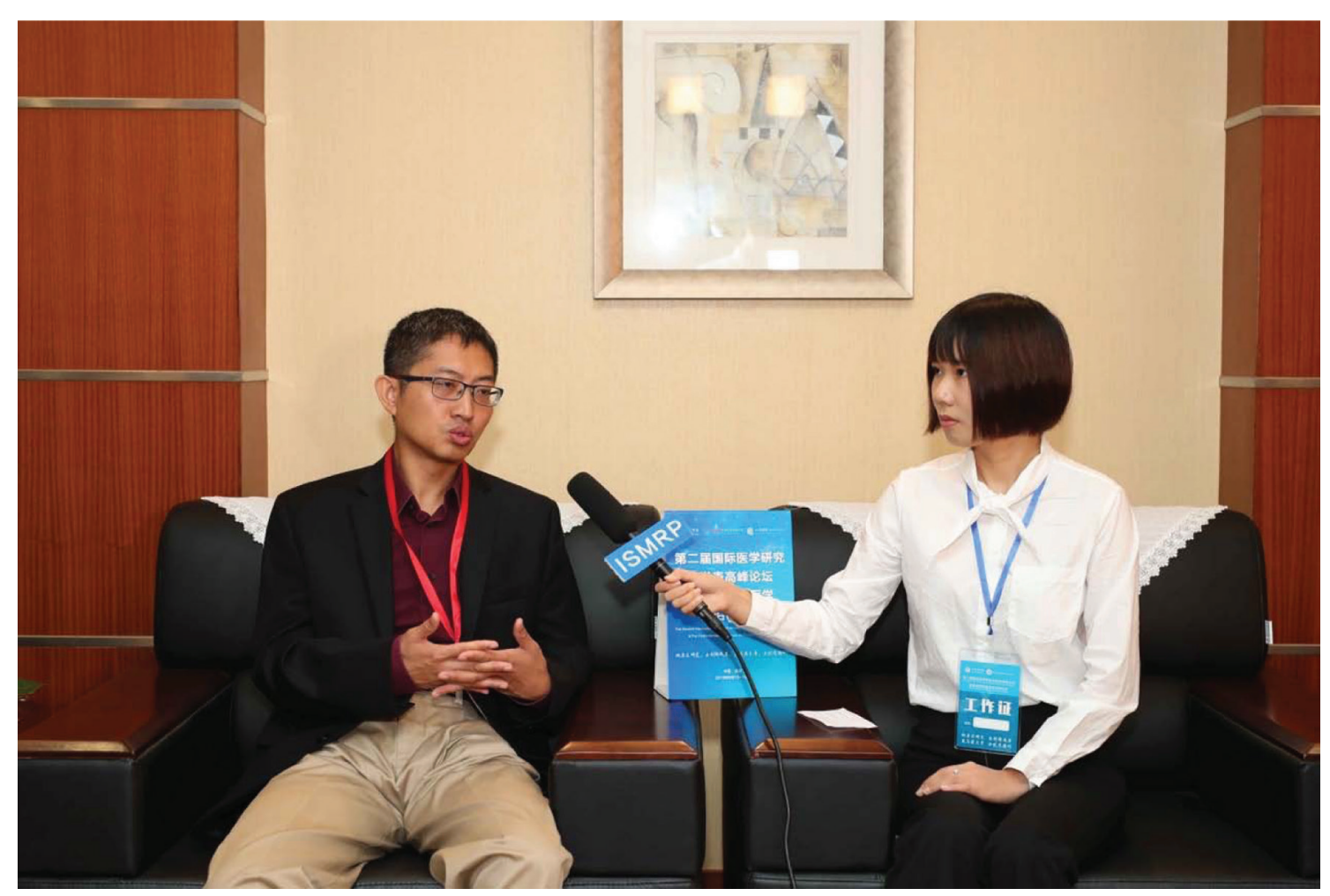

能切实帮助他们如何更好地发文章, 这是我觉得与 很多其他同类型的峰会非常不一样的地方。

本次峰会上, 您的演讲题目是: How
to Publish a High-Impact Research Paper? 您选择这一主题的原因是什么? 希望向听众传递 哪些关键信息?

$A 2$ 对于医生或广大科研工作者, 发文章 这是最基本的一个需求, 但我觉得更重要的是如何 把自己的结果做得更漂亮, 找到更有影响力、更新 颖的科研课题, 这也是标题中的 High-Impact 所要 强调的。从这个角度出发, 再思考如何跟高水平学 术期刊的编辑交流, 跟审稿人交流, 将研究发表在 高影响力的期刊上, 成为一篇具有高影响力的文章, 这可能不是大家常听到的话题。虽然对于广大医生 或科研工作者们而言, 最重要的是解决什么样的临 床问题, 但是后续的与写作发表相关的一些技巧, 也是值得大家关注的。

\section{○2据了解, 您曾担任《自然通讯》(Nature Communications) 科学编辑, 目前担任} 《NEJM 医学前沿》副主编, 都是非常高水平的杂志。 您能否简单介绍一下 《NEJM 医学前沿》杂志的办刊 宗旨? 这对于中国期刊影响力提升有哪些值得借鉴的

经验?

$13 \begin{aligned} & \text { NEJM 医学前沿, 是 New England } \\ & \text { Journal of Medicine 的 中文版, 于 }\end{aligned}$ 2016 年底创刊, 办刊的宗旨是将 New England Journal of Medicine发表的对中国临床工作比较有意义的 一些文章全文翻译成中文, 传递给中国的广大医生 以及对临床医学感兴趣的基础科研的工作者们、学 生以及药厂的科研人员, 实际上性质是以教育为主的 一本杂志。NEJM 医学前沿, 是 New England Journal of Medicine与上海嘉会医学研究和教育集团合 办的, 日后也想以此为基础创办一些立足于中国的、 高水平的、临床医学方面的英文期刊。这样, 将国 内更好水平更高的临床医学的文章带给全世界, 起 到连接世界和中国之间的桥梁作用。

您如何看待中国大量优秀的研究倾向于
发表在国外杂志的现象? 您对于中国目 前在医学研究与发表 (包括期刊建设) 领域的现状有 何思考和期待?

$A 4$ 倾向于将自己最好的文章发表在国外的 一些高水平期刊上, 这个现象的存在是有历史原因 的。以前由于种种原因在中国没有特别好的高水平 学术期刊, 而科研工作者当然希望将自己的科研成 
果发表到好期刊上, 继而让更多的人看到, 所以就 会转而投向国外的一些高水平期刊上, 这是无可厚 非的。不论从对中国的科研事业来说, 还是对全球 的科研事业, 这都是一个正常现象。话虽如此, 但 我认为中国还是应该不断加强科技期刊的建设，而 且目前国家对这方面也有了特别的重视, 不论是从 资金上还是体制上都有新的政策出台。需要强调的 是, 创办一本新的期刊, 需要思考创建这个期刊的 目的是什么。我认为, 创刊目的从根本上来说是为 科研界和医学界服务, 去填补一些空白, 比如, 一 个领域若还没有一个更好的杂志, 那么我们中国可 以创办一本杂志, 把这个领域的空白填补上, 从而 更好地为这一领域的科研工作者医生们服务, 以此 为出发点办刊, 最终也会成功。从长远来看, 如果 我们只是为了办刊而办刊, 这样的期刊可能没有太大 的生命力。 系?

\section{5 这个问题非常深刻, 我主要跟大家分享 \\ 12 下我自己的一些看法吧。医生是否需} 要做科研, 我个人的看法是, 应该取决于医生个人 的兴趣与决定, 作为医生如果对科研感兴趣, 觉得 自己手上有很多很好的结果需要总结出来, 那么当然 应该做科研, 如果只是想成为一名好医生, 对科研 不是特别感兴趣只是被迫做科研, 可能会做出一些 好的结果, 但也可能与我们的初衷背道而驰。现在 出现一些论文被撤稿或数据有问题的现象, 我想很 多时候医生也是出于无奈, 如果有这种出于无奈被 迫的情况, 那么最终的结果也将是大家所不愿看到 的。需要强调一点, 无论做不做科研, 从对患者负 责的角度, 医生应该多阅读文献掌握医学进展中最 前沿的知识。比如我们的临床指南的相关内容及更 新, 2017 年美国对高血压的重新定义, 2018 年召开 的 ESC 对高血压也提出了一个新的指南。这些指南 以及能够改变临床实践的临床试验, 我认为大家不 论是否做科研都应该去读去掌握, 从而更好地为患 者去服务。 